\title{
Conduction Mechanism of 4-Aminoantipyrine as a New Organic Semiconductor
}

\author{
I.S. YAHIA ${ }^{a, b, *, \|}$ \\ ${ }^{a}$ Department of Physics, Faculty of Science, King Khalid University, P.O. Box 9004, Abha, Saudi Arabia \\ ${ }^{b}$ Center of Excellence for Advanced Materials Research, King Khalid University, P.O. Box 9004, Abha, Saudi Arabia \\ (Received September 15, 2012; in final form January 18, 2013) \\ X-ray diffraction pattern of 4-aminoantipyrine was studied and it is a single phase with a polycrystalline \\ structure. 4-aminoantipyrine has hexagonal structure with space group $P 6 / m c c$. The electrical properties of \\ 4-aminoantipyrine were studied in the temperature range $(303-373 \mathrm{~K})$ below the melting point of the studied \\ compound and in the frequency range $(100 \mathrm{~Hz}-100 \mathrm{kHz})$. The obtained results of dc conductivity showed a \\ positive temperature coefficient at the lower temperatures and a negative temperature coefficient at the higher \\ temperatures. The ac conductivity obeys the power law. Ac conductivity can be reasonably interpreted in terms of \\ overlapping-large polaron tunneling model and the correlated barrier hopping model. 4-aminoantipyrine is a good \\ candidate for electronic device due to its electrical conductivity and capacitance.
}

DOI: $10.12693 /$ APhysPolA.125.1167

PACS: 78.40.Me, 78.40.Fy, 72.10.Fk, 77.84.Bw

\section{Introduction}

Organic semiconductors have been extensively studied because of their various electronic and optoelectronic applications. Recently, organic materials are important topics to a wide number of physicists, chemists, and electrical engineering [1-3]. The organic semiconducting materials are grouped as polymer, monomer, and organic compounds. Charge carrier transport properties in organic semiconducting compounds have received considerable attention within the last few decades. Organic semiconductors are used extensively in the fabrication of active and passive solid state devices [4-6].

Electrical conductivity and dielectric measurements at lower frequency in the range $10^{0}-10^{7} \mathrm{~Hz}$ have proven that it is important to gathering a lot of information about the conduction mechanism that dc conductivity alone cannot provide. This technique has been used in many research areas in condensed matter physics, glass science, material science, organic semiconductor and polymer science, etc. This is the major tool for study of ionic conduction in glasses, ceramics, organic and inorganic materials [7-9].

In present communication, the transport properties for 4-aminoantipyrine including dc and ac electrical conductivities were measured at different temperatures and frequencies for the first time. Our work is mainly focused on exploring the conduction mechanism under dc and ac fields for 4-aminoantipyrine as a new organic semiconductor. Overlapping-large polaron tunneling (OLPT) model is the main dominant mechanism over a wide range of

*Permanent address: Nano-Science \& Semiconductor Labs., Physics Department, Faculty of Education, Ain Shams University, Roxy, Cairo, Egypt.

Ile-mail: dr_isyahia@yahoo.com,

ihussein@kku.edu.sa, isyahia@gmail.com temperature. In addition, the sample capacitance was measured at different temperatures and frequencies.

\section{Experimental technique}

4-aminoantipyrine [4-amino-1,2-dihydro-1,5-dimethyl-2-phenyl-3H-pyrazol-3-one] was purchased from Sigma-Aldrich company. The molecular formula of 4-aminoantipyrine is $\mathrm{C}_{11} \mathrm{H}_{13} \mathrm{~N}_{3} \mathrm{O}$ with a molar mass $203.244 \mathrm{~g} / \mathrm{mol}$. Its properties can be described as: pale yellow crystal, its melting point $105-110^{\circ} \mathrm{C}$ and dissolved in water, benzene and ethanol, ethylether [10]. The molecular structure of 4 -aminoantipyrine is shown in Fig. 1.<smiles>Cc1c(N)c(=O)n(-c2ccccc2)n1C</smiles>

Fig. 1. Molecular structure of 4-aminoantipyrine.

X-ray diffraction pattern (XRD) was obtained from a modern automated and computerized diffractometer (X'pert) utilized monochromatic $\mathrm{Cu} K_{\alpha}$ radiation source $(\lambda=1.541 \AA)$ operated at $40 \mathrm{kV}$ and $30 \mathrm{~mA}$. The XRD pattern was recorded automatically in the $2 \theta$ range $4-80^{\circ}$ with scanning step $0.4 \mathrm{~s}$ and step size 0.02 .

In order to measure both direct (dc) and alternating (ac) current conductivities, the studied sample was shaped in the form of pellet by applying 5 ton using hydraulic pressure. The pellet has a thickness $0.5 \mathrm{~mm}$ and radius $13 \mathrm{~mm}$. The dc electrical resistance $R$ for the studied sample was measured automatically by using a digital electrometer (Keithley type E 616 A). A homemade standard cell made from brass and teflon (to isolate the lower brass contact) was used to connect the upper and lower 
parts of the sample under investigation. A movable upper electrode was adjusted and screwed until the sample became in a good contact between the two electrodes of the holder without using any metallic pastes i.e. to avoid the penetration of the paste inside the organic samples.

For ac measurements, programmable automatic RLC bridge (PM 6304 Philips) was used to measure directly the impedance $(Z)$ and the capacitance $(C)$ of the studied sample using the same holder as mentioned before. The studied sample is represented on the screen of RLC bridge by a resistance $(R)$ connected in parallel with a capacitance $(C)$. The sample holder was placed and fixed in a high shielded furnace. Heating of the sample was controlled by a calibrated Chromel-Alumel thermocouple connected to a temperature controller closer to the sample [11, 12].

\section{Results and discussion}

\subsection{X-ray diffraction pattern of 4-aminoantipyrine}

X-ray diffraction (XRD) pattern of 4-aminoantipyrine is shown in Fig. 2. It is clear that 4-aminoantipyrine is a single phase with a polycrystalline structure. Indexing and refinement of the XRD data were performed using Crysfire and Checkcell software, respectively [13, 14]. Throughout our calculation, the strongest diffraction lines with intensity larger than $10 \%$ corresponding to the highest peak intensity on the XRD pattern were chosen. The calculated Miller indices $h k l$ of the diffraction lines are shown in Fig. 2. 4-aminoantipyrine has hexagonal structure and the calculated unit cell parameters are $a=22.5375 \AA, b=22.5375 \AA, c=5.8497 \AA, \alpha=90^{\circ}$, $\beta=90^{\circ}$ and $\gamma=120^{\circ}$ with space group $P 6 / m c c[13,14]$.

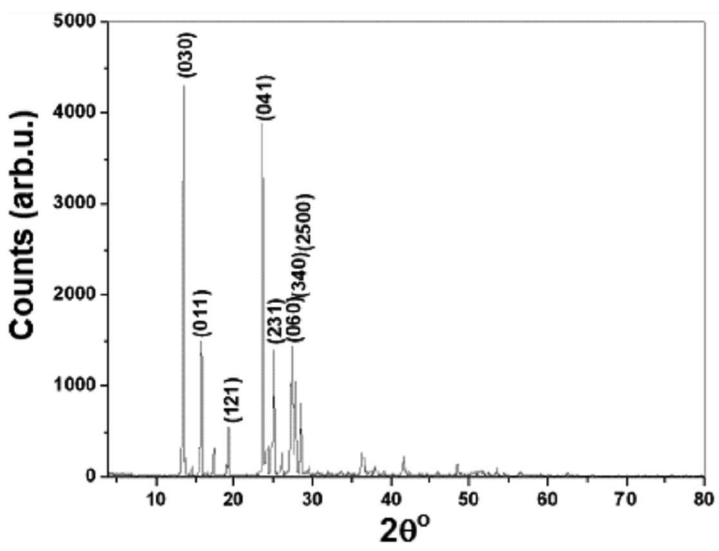

Fig. 2. XRD pattern of 4-aminoantipyrine in powder form.

\subsection{Temperature dependence of dc electrical conductivity}

Dc electrical conductivity for 4-aminoantipyrine was calculated according to the well known Arrhenius equation as follows [11, 12]:

$$
\sigma_{\mathrm{dc}}=\sigma_{0} \exp \left(\frac{-\Delta E_{\sigma}}{k_{\mathrm{B}} T}\right),
$$

where $\Delta E_{\sigma}$ is the electrical activation energy, $T$ is the

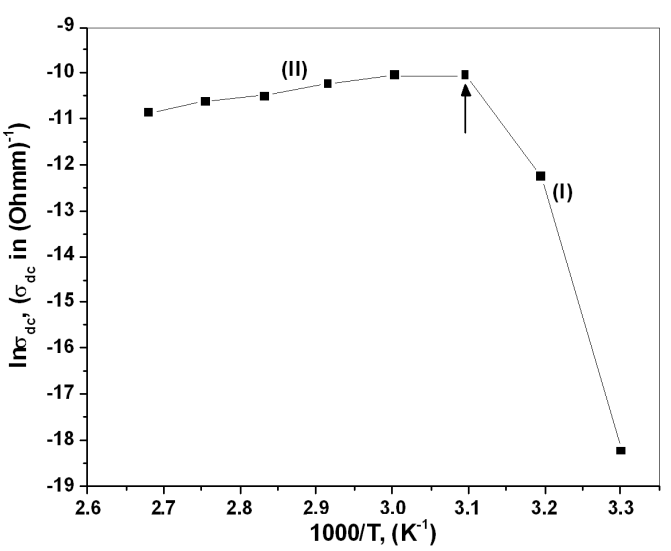

Fig. 3. Plotting of $\ln \sigma_{\mathrm{dc}}$ versus $1000 / T$ for 4 -aminoantipyrine.

absolute temperature, $k_{\mathrm{B}}$ is the Boltzmann constant and $\sigma_{0}$ is the pre-exponential factor.

Plotting of $\ln \sigma_{\mathrm{dc}}$ versus $1000 / T$ for 4-aminoantipyrine is shown in Fig. 3 in the temperature range (303-373 K) below the melting point of the studied compound. It is clear that the dc electrical conductivity increases rapidly with increasing temperature up to $323 \mathrm{~K}$ followed by a decrease in its value up to $373 \mathrm{~K}$. Also, it is clear that the dc conductivity increases with about eight orders of magnitude through a small range of temperature. This response is expected to be effective in electronic devices under zero frequency. So, there are two distinct regions i.e. region (I) with positive activation energy and region (2) with negative activation energy. Our explanation can be understood for both regions as follows: (1) For region (I), the curve shows a positive temperature coefficient (PTC) of the conductivity and this behaviour is like the semiconductor behaviour [15-17] i.e. the electrical conductivity increases with increasing temperature. The observed increase in the conductivity with temperature can be associated with the number of $\pi$-electrons inside the organic compound, when the activation energy may also be related to electron activation within the structure. The $\pi$-electron mobility and the semiconductor behaviour of 4-aminoantipyrine result from the transfer of $\pi$-electrons [6]. (2) For region (II), it is found that the electrical conductivity is decreased with further increase of temperature i.e. the curve shows a negative temperature coefficient (NTC). The decrease of conductivity can be explained by the scattering of carriers by phonons due to lattice vibrations at this range of temperature [15-17]. The slope of this region depends on the nature and structure of the sample [15, 17]. The phenomena of discontinuation observed in the electrical conductivitytemperature relationship showed one or two regions with positive and negative activation energies, probably due to presence of phase transition [18]. This behaviour was reported before in other organic materials as reported by Yakuphanoglu et al. [17, 19] for aqua[bis(2-dimethylaminomethyl-4-NITphenolato)] copper(II) and $\mathrm{Ni}\left(\mathrm{L}^{1} \mathrm{H}_{2}\right)$ as a typical example for our situation. 


\subsection{Temperature and frequency dependences of ac electrical conductivity}

For crystalline, polycrystalline and amorphous materials, the total electrical conductivity at a particular frequency $(\omega)$ and temperature consists of two components (dc and ac) as follows [20-23]:

$$
\sigma_{\text {tot }}(\omega)=\sigma_{\mathrm{ac}}(\omega)+\sigma_{\mathrm{dc}}(\omega=0),
$$

where $\sigma_{\mathrm{dc}}(\omega=0)$ is the dc electrical conductivity and $\sigma_{\mathrm{ac}}(\omega)$ is the ac electrical conductivity. It is assumed that the $\mathrm{dc}$ and ac conductivities arise from completely different processes [24, 25]. The main distinguishing feature of ac conduction as compared with dc conductivity is that for ac, the only necessary is to transfer an electron between a pair of states, whereas in dc, a continuous percolation path between the electrode is necessary for current to flow $[24,25]$. This strongly suggests the following approach, which is adopted in calculations of ac loss, namely to consider the polarizability on an isolated pair of states and to sum up the losses due to such pairs to obtain overall response of the whole sample $[24,25]$.

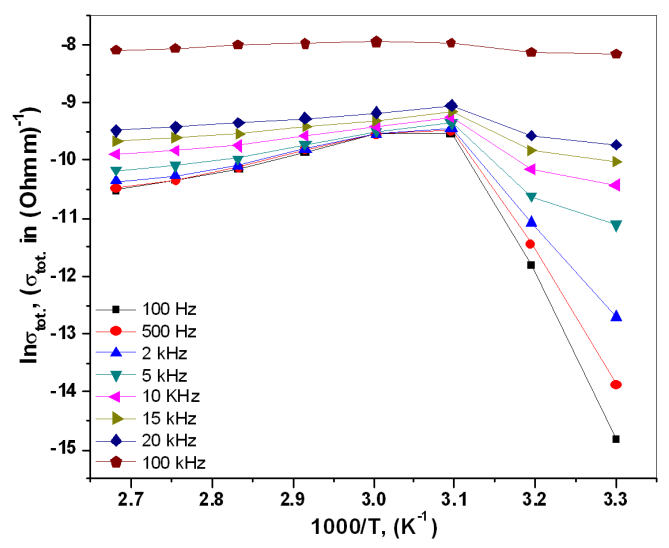

Fig. 4. Temperature dependence of $\sigma_{\text {tot }}(\omega)$ for 4 -aminoantipyrine at different frequencies.

The frequency dependence of the total electrical conductivity $\sigma_{\text {tot }}(\omega)$ at different temperatures is shown in Fig. 4. It can be observed from this figure that $\sigma_{\text {tot }}(\omega)$ increases with increasing temperature up to $323 \mathrm{~K}$ followed by a decrease of its value up to $373 \mathrm{~K}$. The frequency enhances the conductivity changes of 4 -aminoantipyrine by seven order of magnitudes at $100 \mathrm{kHz}$. This response is very effective in the electronic devices under the applied frequency. For ac conductivity, Eq. (2) can be rewritten as [20-23]:

$$
\sigma_{\mathrm{ac}}(\omega)=\sigma_{\text {tot }}(\omega)-\sigma_{\mathrm{dc}}(\omega=0)=A \omega^{s},
$$

where $A$ is a pre-exponential factor and $s$ is the frequency exponent, which in general is less than 1 [17]. The temperature dependence of ac electrical conductivity $\sigma_{\text {ac }}(\omega)$ at different frequencies has the same profile of $\sigma_{\text {tot }}(\omega)$. Plotting of $\ln \sigma_{\mathrm{ac}}(\omega)$ versus $\ln \omega$ is shown in Fig. 5 for the studied organic material. It is clear that the plot has linear plots after a certain point i.e. the conductivity increases linearly at the higher values of the applied frequencies. At lower values of frequencies, it is evident that there is a dc contribution to the ac conductivity [17]. Such dependence can be explained by the variable range hopping (VRH) mechanism. The VRH model is frequency independent and only weakly temperature dependent compared to band theory. Such behaviour was described previously in other organic materials [17].

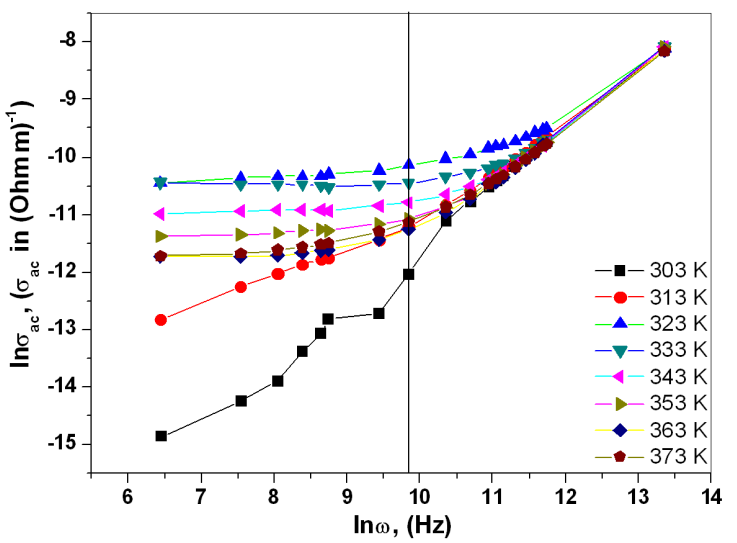

Fig. 5. Frequency dependence of $\sigma_{\mathrm{ac}}(\omega)$ for 4-aminoantipyrine at different temperature.

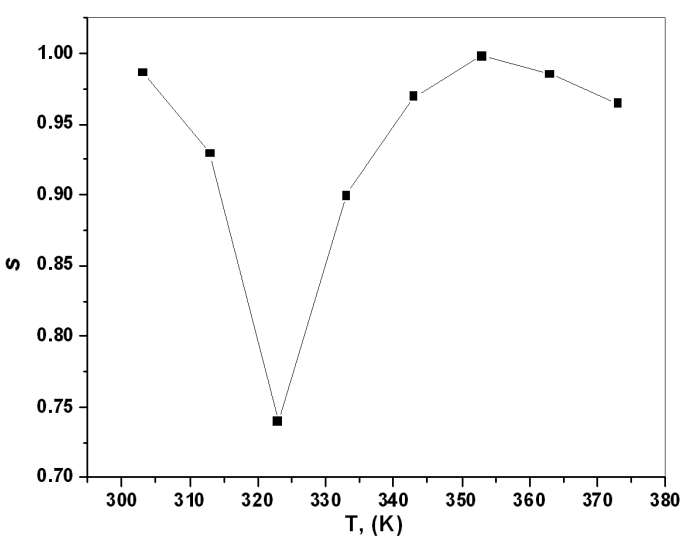

Fig. 6. Temperature dependence of the frequency exponent $s$ for 4-aminoantipyrine.

Values of the frequency exponent $s$ were calculated from the slopes of linear parts of Fig. 5 at the higher frequencies. Figure 6 represents the plot of the frequency exponent $s$ at different temperatures for 4-aminoantipyrine. It is clear that the frequency exponent $s$ exhibits two distinct regions as follows: (1) Region (I) in which the values of $s$ decreased with increase of temperature to reached its minimum values at $323 \mathrm{~K}$ followed by an increase in its values up to $353 \mathrm{~K}$. (2) Region (II) in which the values of $s$ is decreased with increase of temperature up to $373 \mathrm{~K}$. Many different models can be used to describe the conduction mechanism of different materials (organic, inorganic, chalcogenide, ceramics, etc.) under the applied ac field. It is generally assumed that the pair 
approximation i.e. the dielectric loss occurred due to the carrier motion to be localized within pairs of sites. Two distinct relaxation processes, namely quantum mechanical tunneling and classical hopping over barrier or some combinations between them, describe the ac conduction as follows $[11,12]$ :

(i) For quantum mechanical tunneling (QMT) model, the frequency exponent $s$ is almost equal/around the derived value 0.8 and increases slightly with increasing temperature or independent of temperature. Accordingly, QMT model cannot be considered to describe the obtained results [26].

(ii) For the non-overlapping small polarons (NSPT) model, the exponent $s$ is temperature dependent and increased with increasing temperature. Therefore, NSPT model cannot be applied to the obtained results [21].

(iii) For OLPT model, the frequency exponent $s$ is both temperature and frequency dependent. Also, $s$ decreases with increasing temperature to a minimum value at a certain temperature then it continues to increase with increasing temperature. Therefore, OLPT model supports the obtained results for region (I) [24].

(iv) For the correlated barrier hopping ( $\mathrm{CBH})$ model, the values of the frequency exponent $s$ are decreased with increasing temperature. This is in good agreement with the obtained results for region (II) at the higher temperature [27-30].

\subsection{Temperature and frequency dependences of the measured capacitance $C$}

The temperature dependence of the measured capacitance at different frequencies for 4-aminoantipyrine is shown in Fig. 7. It is clear that the measured capacitance is rapidly increased up with temperature until reached its maximum at $333 \mathrm{~K}$ (an inversion point) followed by gradually decreased in its values with further increased of the temperature up to $373 \mathrm{~K}$. Also, the values of measured capacitance are higher at the lower frequency $(100 \mathrm{~Hz})$ and reached its lower values at high frequencies $(100 \mathrm{kHz})$. It is obvious that there is compatibility between the measured capacitance profiles and both $\mathrm{dc}$ and ac electrical conductivities. The temperature dependence of the measured capacitance can be described by Goswami and Goswami model as described by the following equation [31]:

$$
C=C_{\infty}+\frac{1}{\omega^{2} R^{2} C_{\infty}},
$$

where $R$ is the sample resistance, $\omega=2 \pi f$ is the angular frequency, $f$ is the applied frequency and $C_{\infty}$ is the capacitance at the higher frequency. According to Eq. (4), the increase of capacitance $C$ with temperature may be due to decrease of the sample resistance $R$ with temperature [32]. This behavior can be attributed to the effect of charge redistribution by mean carrier hopping on defects [33-35]. At the lower frequency, the charge on the defects can be rapidly redistributed, so that defects closer to the positive side of the applied field become negatively charged, while defects closer to the negative side of the applied field become positively charged. This leads to a screening of the field and an overall reduction in the electrical field because capacitance is inversely proportional to the field and this leads to the increasing capacitance [33-35]. This reduction in the field for a given voltage results in the increased capacitance observed as the frequency is lowered. At the higher frequency, the defects no longer have enough time to rearrange inversions to the applied voltage; hence, the capacitance decreases to their its minimum value $C_{\infty}[33-35]$. The temperature is more effective on the carrier hopping because of the increase in the thermal emission of charges.

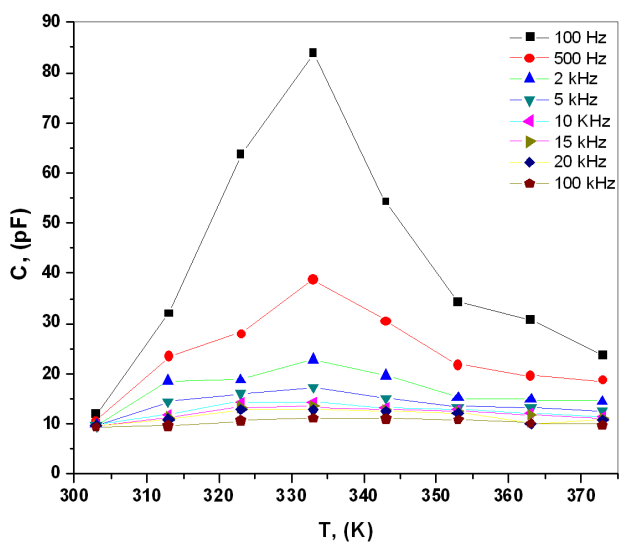

Fig. 7. Variation of the measured capacitance $C$ with temperature at different frequencies for 4-aminoantipyrine.

\section{Conclusion}

4-aminoantipyrine has hexagonal structure and the calculated unit cell parameters are $a=22.5375 \AA, b=$ $22.5375 \AA, c=5.8497 \AA, \alpha=90^{\circ}, \beta=90^{\circ}$ and $\gamma=120^{\circ}$ with space group $P 6 / m c c$. The obtained results of $\mathrm{dc}$ conductivity for 4-aminoantipyrine showed the semiconductor behavior in low temperatures as it has a PTC. At the higher temperatures, it showed an TC. The ac conductivity is found to obey the power law $\sigma_{\mathrm{ac}}(\omega)=B \omega^{s}$ and the frequency exponent $s$ is decreased with increasing temperature to reach its minimum values at $323 \mathrm{~K}$ followed by an increase in its values up to $353 \mathrm{~K}$ then it is decreased with increasing temperature up to $373 \mathrm{~K}$. The temperature dependence of ac conductivity can be reasonably interpreted in terms of OLPT model in low temperatures and the $\mathrm{CBH}$ model at high temperatures. The capacitance was found to decrease with increasing frequency and to increase with increasing temperature reaching to a maximum then decease. This behavior is described by Goswami and the Goswami model. 


\section{References}

[1] F. Yakuphanoglu, Physica B 393, 139 (2007).

[2] T.A. Skotheim, R.L. Elsenbaumer, J.R. Reynolds, Handbook of Conducting Polymers, Marcel Dekker, New York 1998.

[3] M.C. Petty, M.R. Bryce, D. Bloor, An Introduction to Molecular Electronics, Edward Arnold, London 1995.

[4] L. Leontie, M. Roman, I. Caplanus, G.I. Rusu, Prog. Org. Coat. 44, 287 (2002).

[5] G. Liang, T. Cui, K. Varahramyan, Microelectron. Eng. 65, 279 (2003).

[6] F. Yakuphanoglu, I. Erol, Physica B 352, 378 (2004).

[7] C.J.F. Boltcher, P. Bordewijk, Theory of Electric Polarization, Vols. I and II, 2nd ed., Elsevier, New York 1978.

[8] N.G. McCrum, B.E. Readond, G. Williams, An Elas tic and Dielectric Effects in Polymeric Solids, Wiley, New York 1967.

[9] Komilla Suri, S. Annapoorni, R.P. Tandon, J. NonCrystalline Solids 332, 279 (2003).

[10] www.chemyq.com/En/xz/xz1/4750nlimc.htm .

[11] Sh.A. Mansour, I.S. Yahia, F. Yakuphanoglu, Dyes Pigments 87, 144 (2010).

[12] Sh.A. Mansour, I.S. Yahia, G.B. Sakr, Solid State Commun. 150, 1386 (2010).

[13] A.A.M. Farag, I.S. Yahia, Synth. Metals 161, 32 (2011).

[14] I.S. Yahia, M.S. Abd El-Sadek, F. Yakuphanoglu, Dyes Pigments 93, 1434 (2012).

[15] F. Yakuphanoglu, Ph.D Thesis, Firat University, Elazig, Turkey 2002.

[16] U. Schatzschneider, T. Weyhermüller, E. Rentschler, Eur. J. Inorg. Chem., 2569 (2001).

[17] F. Yakuphanoglu, Y. Aydogdu, U. Schatzschneider, E. Rentschler, Solid State Commun. 128, 63 (2003).

[18] M.S. Masoud, S.A. El-Enein, E. El-Shereafy, J. Therm. Anal. 37, 365 (1991).

[19] Y. Aydogdu, F. Yakuphanoglu, A. Aydogdu, E. Tas, A. Cukurovali, Solid State Sci. 4, 879 (2002).

[20] A. Ghosh, Phys. Rev. B 41, 1480 (1990).

[21] A. Ghosh, Phys. Rev. B 42, 5665 (1990).

[22] M. Pollak, G.E. Pike, Phys. Rev. Lett. 28, 1494 (1972).

[23] M. Pollak, T.H. Geballe, Phys. Rev. 122, 1742 (1961).

[24] A.R. Long, Adv. Phys. 31, 553 (1982).

[25] A.R. Long, N. Balkan, W.R. Hogg, R.P. Ferrier, Philos. Mag. B 45, 497 (1982).

[26] A. Ghosh, Phys. Rev. B 41, 1479 (1990).

[27] G.E. Pike, Phys. Rev. B 6, 1572 (1972).

[28] S.R. Elliott, Philos. Mag. B 37, 135 (1978).

[29] S.R. Elliott, Philos. Mag. B 36, 129 (1978).

[30] B.K. Chaudhuri, K. Chaudhuri, K.K. Som, J. Phys. Chem. Solids 50, 1147 (1989).

[31] A. Goswami, A.P. Goswami, Thin Solid Films 16, 175 (1973).

[32] M.A.M. Seyam, Appl. Surf. Sci. 181, 128 (2001).
[33] P.W. Zukowski, S.B. Kantorow, D. Maczka, V.F. Stelmakh, Phys. Status Solidi A 112, 695 (1989).

[34] A. Vasudevan, S. Carin, M.R. Melloch, E.S. Harmon, Appl. Phys. Lett. 73, 671 (1998).

[35] A.M.A. El-Barry, H.E. Atyia, Physica B 368, 1 (2005). 